\title{
SPECIATION OF ORGANOMERCURIALS IN MARINE SAMPLES USING CAPILLARY ELECTROPHORESIS
}

I. Medina

Institute of Marine Research, C.S.I.C., Vigo, Spain

E. Rubí, M. C. Mejuto and R. Cela*

Department of Analytical Chemistry, Faculty of Chemistry, University of Santiago de Compostela, Santiago, Spain

*Author for correspondence.

Summary-A rapid method for speciation and determination of organomercury compounds in biological samples of marine origin using Capillary Electrophoresis (CE) is reported. Organomercurials were extracted from the samples by means of the classical Westöö procedure thus giving organomercury-cysteine complexes which can be separated from each other by means of CE resulting in effective speciation. Electrophoretic separation was achieved in an open silica capillary tube at 15-18 kV using a 100mM sodium borate buffer ( $\mathrm{pH}$ 8.35). All mercury species were distinctively separated within $12 \mathrm{~min}$. Results are presented for the analysis of real marine samples and reference materials, and compared with those obtained by the GC commonly accepted procedure.

Many studies ${ }^{1}$ have reported that organic mercury forms, especially the methylated forms, are considerably more toxic than the inorganic ones. In fact, methylmercury is considered to be one of the most dangerous chemical species in the environment because of its intrinsic toxicity and availability to living organisms. However, the analytical measurement and speciation of organomercurials in complex natural world samples remains a very difficult problem with two main requirements: high selectivity and very low detection limits. Moreover, since high levels of methylmercury have been found in various marine organisms, especially in large predators (tuna, swordfish, etc.) analytical methodology for methylmercury must be at the same time fast, low in cost and reliable for use in the routine analysis of biological and environmental samples.

During the last few decades several procedures have been proposed for these purpose $\mathrm{e}^{2-15}$ 
but the most frequently used approach is the gas chromatography separation of the halogen derivatives of organomercurials with electron capture detection ${ }^{4,16-20}$ based on the classical Westöö's method ${ }^{4}$. On the other hand, several papers ${ }^{17,21-23}$ have focused on the drawbacks of these gas chromatographic procedures. In fact, the lack of consistent reproducibility, the tailing of peaks, the absorption of variable amounts of the organomercurial compounds in the GC-system and the need of special column conditioning or priming with inorganic mercury salts and related consequences in the analysis of real samples (dead times of operation, doubts about the real nature of the compound detected after the priming, frequent drop-out of the peaks, etc.) as well as in the life of the columns and detector itself, has led to search for alternative procedures to carry out the organomercurials analysis on a more reliable basis. Recently ${ }^{24}$ some of these drawbacks have been overcome by means of the use of special narrow bore chromatographic columns.

Capillary electrophoresis has demonstrated in recent years powerful capabilities for the resolution and analysis of very complex samples. ${ }^{25}$ Although several papers ${ }^{26}$ were dealing with the separation of inorganic ions and neutral or charged complexes of metals, this technique had so far limited application for speciation studies. ${ }^{27}$ The high resolution and peak capacity characteristics of this technique could be used favourably in speciation studies for very complex samples. However, since the time needed for separation is usually very short in this technique, having full possibilities in automation, it can be easily used for routine analysis.

In this paper, a procedure is presented for speciation of organomercurials and the quantitative determination of methylmercury in marine samples by means of capillary electrophoresis. Cysteine has been used to complex organomercurials according to the classical extraction method of Westöö ${ }^{4}$. The resulting cysteine-organomercury complexes were well separated in an electric charge density, using an alkaline buffer. Parameters affecting the separation have been optimized and the results for some types of marine samples as well as for an existing reference material has been compared with those obtained by the usual gas chromatographic method. ${ }^{19}$

\section{EXPERIMENTAL}


Reagents

All reagents used were HPLC or analytical grade. Ultrapure water was obtained by means of a MilliQ system (Millipore Co.). Mercury(II) chloride, methyl, phenyl and ethyl-mercury chlorides (Merck) were used as calibrants. A stock solution (1.00 g/l.) in methanol was used to obtain weekly standards by suitable dilution with methanol: water (1:1). All the organomercury solutions were protected against light and stored at $4^{\circ}$ in the dark when not in use. Aqueous cysteine chlorhydrate was used instead of cysteine acetate solution recommended by Westöö. ${ }^{4}$ Buffers were prepared with boric acid and sodium hydroxide to $\mathrm{pH}$ 8.35. Aqueous cysteine and the buffer containing sodium dodecyl sulfate were prepared weekly and stored at $4^{\circ}$ when not in use.

Standards

Standards for injection containing one or more organomercurials were prepared by mixing $400 \mu 1$ of the appropriate standard solution of each organomercurial and $100 \mu 1$ of $1 \%$ aqueous cysteine solution.

Preparation of samples

Samples were prepared in the way outlined in the scheme of Fig. 1 which essentially agrees with the Westöö procedure. ${ }^{4}$ Final aqueous extracts were subjected to capillary electrophoresis separation.

Capillary electrophoresis

High performance capillary electrophoresis was carried out at $30^{\circ}$ in a $75-\mu m$ (i.d.) 50 cm long fused silica capillary (window at $5 \mathrm{~cm}$ ) using a PACE system 2050 (Beckman). Samples were introduced into the capillary by pressure injection over 8.4 see (corresponding to a volume of $77.4 \mathrm{nl}$ ). Electroendosmosis was toward the cathode at 18 $\mathrm{kV}$ in a $100 \mathrm{mM}$ sodium Borate buffer (pH 8.35) during $12 \mathrm{~min}$. UV detection at $200 \mathrm{~nm}$ and a System Gold (Beckman) equipment were used for detection, data acquisition and processing. 
The capillary was daily prepared by flushing with $0.5 \mathrm{M}$ sodium hydroxide for $10 \mathrm{~min}$, followed by a 10-min rinse with ultrapure water. A typical run consisted of a 2-min washing period with ultrapure water, followed by 3 min of capillary reconditioning with the selected buffer.

\section{RESULTS AND DISCUSSION}

\section{Buffer selection}

The $\mathrm{pH}$ of the buffer used to carry out the electrophoregram, was usually the most important operational parameter. For cysteine, the isoelectric point was 6.24 thus, in alkaline media, complexes formed by cysteine with organomercurials were charged negatively. As can be seen in Fig 2(a), using a 100mM borate buffer (PH 8.35) it was possible to separate the cysteine-methylmercury complex from the excess of ligand. Also a small peak having a retention time lower than the one of the complex was observed. Since the standards for injection were prepared simply by mixing adequate solutions of methylmercury and cysteine just before the injection it was possible that this small peak would correspond to the not complexed form neutral methylmercury. This hypothesis was confirmed by comparison with the behaviour of methylmercury alone (no cysteine added) as shown in Fig. 2(b). These data were in accordance with the electroendosmosis theory for alkaline media. In the same way the elution order of the complex and the free ligand form were according to the number of negative charges corresponding to the molecules:<smiles>CSCC(C)C(=O)O[Sb]</smiles>

It was possible to obtain separations using media of $\mathrm{pH}$ lower than the isoelectric point. Experiments were carried out using a phosphate buffer ( $\mathrm{pH} 2.5)$. However, as shown in Fig. 3, the peaks obtained were much less intense. In fact, the electrophoregram in Fig. 3 corresponds to the same standard solution as the one in Fig. 2 but the absorbance scale is 20 times greater. In this case the use of acidic buffers resulted in poor sensitivity.

The use of the borate buffer ( $\mathrm{pH}$ 8.35) enabled the separation of the three organomercurials to be achieved as shown in Fig. 4. In alkaline media the (1:2) 
mercury(II)-cysteine complex had two negative charges which meant that inorganic mercury appeared to the right of the free cysteine peak in the electrophoregram. The order of elution was ethyl, methyl and phenylmercury. Decreasing the applied potential (up to $10 \mathrm{kV}$ ) did not improve the resolution of ethyl-methyl mercury pair. For phenylmercury, the contribution of the aromatic ring to the absorbance led to a greater sensitivity as compared to the others.

The influence of variable concentrations of a micellar agent [sodium dodecyl sulphate (SDS)] in the borate buffer was also studied, trying to improve the resolution of the ethyl-methylmercury pair. However this possibility was discarded owing to the fact that the peaks appeared broader and with lower area values while no significant improvement in overall resolution was achieved. In Fig. 5 it can be seen that the methylethyl pair was somewhat better resolved but in this case a significant overlap between methyl and phenyl peaks took place. Moreover, from a practical point of view, the inclusion of SDS into the buffer meant that it could not be used for more than 2-3 days. On the contrary a simple borate buffer remained usable during weeks.

Signal linearity

The relationship between the amount of methylmercury injected and peak area in the electrophoregram showed good linearity (correlation coefficient 0.9974 , R-squared $=$ $99.48 \%$ with a standard error of estimate of $4.39 \times 10^{-3}$ ) in the range $12-300 \mathrm{pg}$. The detection limit was $10 \mathrm{pg}$ in the conditions described in the Experimental section for a signal to noise ratio of 10:1. Data for repeatability (retention times and peak areas) for five successive injections of a methylmercury standard (11.2 ng injected) were: average retention time $=4.28 \pm 0.01$ seconds; variation coefficient $=0.3 \%$ and, average peak area $($ arbitrary units $)=4.76 \pm 0.10$; variation coefficient $=2.1 \%$. These data also indicated that the procedure sequence (programmed prerinse, etc.) were adequate for practical purposes. In Table 1 some data of reproducibility for several standard solutions of methylmercury determined in different days (one measurement each day) are listed. It is observed that variation coefficients were very similar with those of repeated injections, hence the ruggedness of the procedure was considered to be good. 
Table 2 summarizes the results for recovery of methylmercury along the proposed procedure. For these experiments a freeze dried mussel tissue sample was used. This sample did not give any detectable signals for methylmercury nor for inorganic mercury so, it was considered as a matrix-blank sample. A 1.00-g sample of this material was used in the experiments adding variable amounts of a methylmercury standard in water, Once homogenized and dried, the resulting material was analyzed by means of the proposed procedure. Simultaneously the same standard solutions used for spiking the mussel sample were extracted and analyzed. Thus the only difference between the rows in Table 2 labelled as "sample" and those labelled as "blank" is the presence or absence of the biological matrix. The results in Table 2 indicate that important losses took place during the extraction process. Only $72 \%$ of the methylmercury initially present is extracted. Furthermore, the results of a two-way ANOVA carried out on the data in Table 2 indicated a significant effect of the matrix in the methylmercury recovery. This effect can be explained by considering the formation of persistent scums in the extraction stages which hampered correct phase separation. Taking this into account, the recovery for methylmercury in real samples by the proposed procedure was $64.7 \pm$ $3.1 \%$.

Analysis of real samples

The initial experiments with real samples were carried out using a 1\% cysteine acetate solution as back-extractant, because this is the usual concentration used in the gas chromatographic method. ${ }^{4}$ Soon, it was observed that this excess of cysteine hampered the effective separation of the methylmercury complex from the free ligand when methylmercury concentration in the samples was low. This effect was provocated not only by the relative size of both peaks but it is believed that the excess of free ligand blocked-out or saturated the walls of the capillary tube giving poor separations. After a series of experiments, it was demonstrated that the use of a $0.1 \%$ solution of cysteine chlorhydrate allowed to complexation of all the organomercurials in the samples without impairing the peak separation.

It was noted that one artifact occurred when analyzing real samples. This consisted of a gross peak at a retention time lower than the corresponding one for methylmercury. As 
shown in Figs 6 and 7, this peak did not overlap with that of methylmercury, so in most cases it was not necessary to take any special precaution. In any case, this unknown compound has a lifetime of about $10 \mathrm{hr}$, so, if the extracts were left to stand for some hours in a dark cool place before the analysis, the artifact peak almost disappeared (see Fig. 7) enabling a more easy and accurate integration of the methylmercury peak which area did not change in this time. Although we have observed this artifact in all the real samples analyzed, we are not sure that it was present in other types of samples. Table 3 summarizes some of the results obtained for real samples using the proposed procedure. These results are compared in this table with the ones obtained by means of the usual gas chromatographic procedure ${ }^{19}$ for the same samples. It can be seen that the results for CE analyses were systematically lower than those obtained by gas chromatography for which no cysteine back-extraction was carried out. This is because of the aforementioned losses in the back-extraction stage with cysteine. When the results for CE analyses were corrected for these losses (last column in Table 3) the obtained data were in good agreement with the gas chromatographic ones as well as with certified values for the reference material analysed.

\section{CONCLUSIONS}

Up to this date, capillary electrophoresis has been only used in a very limited extent in speciation studies. The presented results shows that this technique could be used as a powerful tool in the separation of several organometallic forms in biological and environmental samples. Even more, separations could be understood on the basis of a well-established background without the need for special or sophisticated separation schemes. In the particular case of organomercurials speciation, the use of capillary electrophoresis avoids the drawbacks associated with gas chromatographic procedures and is rapid, and simple enough to be used on a routine basis.

Acknowledgements- A part of this work has been supported by the Spanish Interministerial Commission for science and Technology (CICYT) under the project ALI-0773.

\section{REPERRNCES}


1. IPCS. Environmental Health Criteria 101: Methyl-mercury, World Health

Organization, Geneva, 1990.

2. J. F. Uthe and F. A. J. Armstrong, in L. Frieberg and J. Vostal (eds), Mercury in the Environment, pp. 21-53. CRC Press, Cleveland, OH, 1972.

3. J. C. Cage, Analyst, 1961, 86, 457.

4. G. Westöö, Acta Chem Scand., 1966, 20, 2131; 1967, 21, 1790; 1968, 22, 2277.

5. D. Beauchemin, K. W. M. Siu and S. S. Berman, Anal. Chem., 1988, 60, 2587.

6. S. S. Berman, K. W. M. Siu, P.S. Maxwell, D. Beauchemin and V. P. Clancy, Z.

Ana1. Chem., 1989, 333, 641.

7. L. Magos, Analyst, 1971, 96, 847.

8. I. M. Davies, Anal. Chim. Acta, 1978, 102, 189.

9. M. Fujita and E. Takabatake, Anal Chem., 1983, 55, 454.

10. N. Bloom and W. F. Fitzgerald, Anal. Chim. Acta, 1988, 208, 151.

11, N. Bloom, Can. J. Fish. Aquat. Sci., 1989, 46, 1131.

12. G. Decadt, W. Baeyens, D. Bradley and L. Goeyens, Anal. Chem. 1985, 57, 2788.

13. P. Lansens and W. Baeyens, Anal. Chim. Acta., 1990, 228, 93.

14. W. A. McCreham, Anal. Chem., 1981, 53, 74.

15. O. Evans and O. D. McKee, Analyst, 1987, 112, 983.

16. K. Sumino, Kobe J. Med. Sci., 1968, 14, 115; 1968, 14, 131.

17. S. C. Hight and S. G. Capar, J. Assoc. Off. Anal. Chem., 1983, 66, 1121.

18. G. H. Alvarez, S. C. Hight and S. G. Capar, J. Assoc. Off. Anal. Chem., 1984, 67, 715.

19. S. C. Hight and M. T. Corcoran, ibid., 1987, 70, 24.

20. Mercury(Methyl) in Fish and Shellfish. Rapid Gas Chromatographic Method. First Action, J. Assoc. Off. Anal. Chem., 1988, 71, 210.

21. J. E. O’Reilly, J. Chromatogr., 1982, 23g, 433.

22. E. Nulska, D. C. Baxter and W. Frech, Anal. Chim. Acta, 1991, 249, 5454.

23. E. Rubí, R. A. Lorenzo, C. Casais, A. M. Carro and R. Cela, J. Chromatogr., 1992, 605, 69.

24. R. A. Lorenzo, A. Carro, E. Rubí, C. Casais and R. Cela, J. of AOAC Internat., in press.

25. W. G. Kuhr, Anal. Chem., 1990, 62, R403.

26. W. G. Kuhr and C. A. Moaning, ibid., 1992, 64, 389R

27. M. Albert, C. Demesnay, M. Porthault and J. L. Roca, Analusis 1992, 20, 383. 
Fig. 1. Sample preparation scheme for methylmercury determination.

Fig. 2. Separation of cysteine complexed methylmercury and the cysteine excess by CE. a) Applied potential $20 \mathrm{kV}, 100 \mathrm{mM}$ borate buffer (pH 8.35), 10\% methanol. $13.5 \mu \mathrm{g}$ of methylmercury chloride mixed with $1 \%$ aqueous cysteine, $27 \mathrm{nl}$ injected. b) Same conditions as for a) but an injection of $27 \mathrm{nl}$ of methylmercury chloride standard have been superimposed (dotted line). Key for peaks: (A) methylmercury-cysteine complex; (B) excess cysteine; (C) unknown; (D) uncomplexed methyhnercury.

Fig. 3. Separation of cysteine complexed methylmercury in acidic buffer (100mM phosphate buffer $\mathrm{pH}$ 2.5, 10\% methanol). Applied potential $20 \mathrm{kV}, 13.5 \mu \mathrm{g}$ of methylmercury chloride mixed with $1 \%$ aqueous cysteine. (A) methylmercury peak.

Fig. 4. Speciation of mercmy forms complexed with cysteine, $1.5 \mu$ g methylmercury, $1.5 \mu \mathrm{g}$ ethylmercury, 0.4 . $\mu \mathrm{g}$ phenylmercury and $0.3 \mu \mathrm{g}$ mercury(II) mixed in proportion 1:4 with $0.1 \%$ aqueous cysteine; $27 \mathrm{nl}$ injected. Applied potential $15 \mathrm{kV}$, 100mM borate buffer (pH 8.35), 10\% methanol. (A) ethylmercury; (II) excess cysteine; (C) methylmercury; (D) phenylmercury and (E) inorganic mercury

Fig. 5. Speciation of organomercurials in alkaline buffer containing SDS. Applied potential $15 \mathrm{kV}$. 100mM borate buffer (pH 8.35), 10\% methanol, 25mM SDS. (A) methylmercury; (B) phenylmercury; (C) cysteine excess and (D) ethylmercury

Fig. 6. Analysis of a blank mussel sample spiked with methyl and phenylmercury. Applied potential $15 \mathrm{kV}, 100 \mathrm{mM}$ borate buffer, 10\% methanol. (A) methylmercury; (B) phenylmercury; (C) cysteine excess; (D) unknown

Fig. 7. Analysis of a blank mussel sample spiked with methylmercury (1) injected immediately after extraction and (2) injected after $10 \mathrm{hr}$. Both graphs have been delayed in the time axis to provide a more easy comparison. (A) methylmercury; (B) unknown. game experimental conditions as for Fig. 6 
Table 1. Reproducibility of peak area counts (injections carried out in five different days)

\begin{tabular}{|c|c|c|c|c|c|c|c|}
\hline \multirow[b]{2}{*}{$\begin{array}{l}\text { Methylmercury } \\
\text { amount (pg) }\end{array}$} & \multicolumn{5}{|c|}{ Injection number } & \multirow[b]{2}{*}{ Average } & \multirow[b]{2}{*}{$\begin{array}{l}\text { Variation } \\
\text { Coefficient }\end{array}$} \\
\hline & 1 & 2 & 3 & 4 & 5 & & \\
\hline 193.5 & 0.1808 & 0.1674 & 0.1830 & 0.1712 & 0.1725 & 0.1750 & 3.8 \\
\hline 96.7 & 0.0970 & 0.0907 & 0.0930 & 0.0941 & 0.0959 & 0.0941 & 3.0 \\
\hline 38.7 & 0.0390 & 0.0400 & 0.0410 & 0.0408 & 0.0404 & 0.0403 & 2.0 \\
\hline 30.9 & 0.0260 & 0.0248 & 0.0260 & 0.0266 & 0.0260 & 0.0260 & 2.8 \\
\hline 15.5 & 0.0152 & 0.0157 & 0.0142 & 0.0139 & 0.0150 & 0.0148 & 5.0 \\
\hline 7.7 & 0.0082 & 0.0081 & 0.0085 & 0.0077 & 0.0073 & 0.0079 & 5.9 \\
\hline
\end{tabular}

Table 2. Influence of the matrix in the recovery of methylmercury

\begin{tabular}{lllllllll}
\hline Material* & \multicolumn{2}{l}{$\begin{array}{l}\text { Methylmercury } \\
\text { added (pg) }\end{array}$} & \multicolumn{2}{l}{$\begin{array}{l}\text { Methylmercury } \\
\text { found (pg) }\end{array}$} & $\begin{array}{l}\text { Average } \\
\text { (pg) }\end{array}$ & $\begin{array}{l}\text { Standard } \\
\text { deviation }\end{array}$ & $\begin{array}{l}\text { Variation } \\
\text { coefficient }\end{array}$ & $\begin{array}{l}\text { Recovery, } \\
\%\end{array}$ \\
\hline Mussel & 309.6 & 286.6 & 192.3 & 205.0 & 201.3 & 7.8 & 3.9 & $65.0 \pm 2.5$ \\
Mussel & 154.8 & 102.0 & 107.3 & 102.2 & 103.8 & 3.0 & 2.9 & $67.1 \pm 1.9$ \\
Mussel & 61.9 & 39.9 & 36.2 & 39.0 & 38.4 & 1.9 & 4.9 & $62.0 \pm 3.1$ \\
Blank & 309.6 & 231.8 & 220.2 & 206.3 & 219.4 & 12.7 & 5.8 & $70.8 \pm 4.3$ \\
Blank & 154.8 & 118.1 & 117.3 & 118.3 & 117.9 & 0.5 & 0.5 & $76.1 \pm 0.3$ \\
Blank & 61.9 & 43.5 & 42.0 & 44.5 & 43.3 & 1.2 & 2.8 & $70.0 \pm 2.0$ \\
\hline
\end{tabular}


*Mussel means a freeze-dried mussel tissue sample having a non-detectable level of mercury; blank means the absence of biological material in the experiments

Table 3. Determination of methylmercury in real samples (all results expressed in mercury)

\begin{tabular}{|c|c|c|c|c|c|c|c|}
\hline \multirow[t]{2}{*}{$\begin{array}{l}\text { Sample } \\
\text { material }\end{array}$} & \multicolumn{2}{|c|}{$\begin{array}{l}\text { Methylmercury } \\
\text { content ( } \mu \mathrm{g} / \mathrm{g})\end{array}$} & \multirow[t]{2}{*}{$\begin{array}{l}\text { Sample } \\
\text { Weight } \\
\text { (g) }\end{array}$} & \multirow[t]{2}{*}{$\begin{array}{l}\text { Methylmercury } \\
\text { Injected (pg) } \neq\end{array}$} & \multirow[t]{2}{*}{$\begin{array}{l}\text { Methylmercury } \\
\text { found by CEף } \\
\text { (pg) }\end{array}$} & \multicolumn{2}{|c|}{$\begin{array}{l}\text { Methylmercury } \\
\text { content, corrected } \\
\text { for losses§థ }\end{array}$} \\
\hline & Certified* & Known† & & & & (pg) & $(\mu \mathrm{g} / \mathrm{g})$ \\
\hline DORM-1 & $0.731 \pm 0.060$ & - & 6.0298 & 56.29 & 34.80 & 53.78 & $0.698 \pm 0.025$ \\
\hline DORM-1 & $0.731 \pm 0.060$ & - & 4.0056 & 37.39 & 23.09 & 35.68 & $0.697 \pm 0.033$ \\
\hline MUSSEL & - & $2.142 \pm 0.092$ & 1.0075 & 29.60 & 19.64 & 30.35 & $2.224 \pm 0.084$ \\
\hline COCKLE & - & $1.703 \pm 0.151$ & 1.5441 & 33.59 & 20.88 & 32.27 & $1.636 \pm 0.060$ \\
\hline CLAM & - & $1.585 \pm 0.050$ & 1.4645 & 31.41 & 20.41 & 31.54 & $1.590 \pm 0.081$ \\
\hline TUNA & & $3.662 \pm 0.355$ & 0.9102 & 38.70 & 24.42 & 31.75 & $3.534 \pm 0.344$ \\
\hline
\end{tabular}

*DORM-1 is a Dogfish muscle Certified Reference Material distributed by the National Research Council of Canada.

†Methylmercury concentration values obtained by means of the Hight’s gas chromatographic method ${ }^{19}$ in our laboratory.

$\ddagger$ All the samples were extracted with $40 \mathrm{ml}$ of toluene and backextracted into $5 \mathrm{ml}$ of $0.1 \%$ cysteine colution. Injection volume in all cases were $77.4 \mathrm{nl}$.

$\S$ Assuming a $64.7 \%$ of average recovery in the whole process.

I Average of three independent analyses. 